\title{
Copeptin concentration following cardiac surgery as a prognostic marker of postoperative acute kidney injury: a prospective cohort study
}

\author{
Keigo Yamashita, Takehisa Abe, Yoshihiro Hayata, Tomoaki Hirose, Shun Hiraga, Ryohei Fukuba, \\ Junichi Takemura, Rei Tonomura, Kazuki Yamamoto, Shinya Yokoyama, Shigeki Taniguchi
}

Department of Thoracic and Cardiovascular Surgery, Nara Medical University School of Medicine, Kashihara City, Nara, Japan

Contributions: (I) Conception and design: K Yamashita, S Taniguchi; (II) Administrative support: None; (III) Provision of study materials or patients: None; (IV) Collection and assembly of data: None; (V) Data analysis and interpretation: S Hiraga, R Fukuba, J Takemura; (VI) Manuscript writing: All authors; (VII) Final approval of manuscript: All authors.

Correspondence to: Keigo Yamashita, MD. Department of Thoracic and Cardiovascular Surgery, Nara Medical University School of Medicine, 840 Shijo-cho, Kashihara City, Nara 634-8521, Japan. Email: ke-igo@umin.ac.jp.

\begin{abstract}
Background: Copeptin, the C-terminal portion of the arginine vasopressin precursor, is a novel candidate biomarker. This study investigated the prognostic value of copeptin levels following cardiac surgery for the occurrence of postoperative acute kidney injury.

Methods: We studied 23 patients who underwent cardiac surgery between January 2018 and December 2019. The primary endpoint was postoperative acute kidney injury onset. Copeptin levels were measured before, right after, and daily for 7 days. The patients were divided into two groups according to the copeptin levels: low (values $<43.7 \mathrm{pmol} / \mathrm{L}$ ) and high (values $\geq 43.7 \mathrm{pmol} / \mathrm{L}$ ). Correlations between copeptin levels and variables, such as central venous pressure, were assessed by bivariate analysis.

Results: The high copeptin group exhibited significantly higher levels of arginine vasopressin and cortisol following surgery, compared to those of the low copeptin group. The copeptin concentration following surgery was correlated to central venous pressure $(\mathrm{P}=0.03)$ and norepinephrine administered dose $(\mathrm{P}=0.008)$. Also, the copeptin levels right after surgery robustly predicted the onset of postoperative acute kidney injury (area under the receiver operating characteristic curve of $0.83, \mathrm{P}=0.004$ ).

Conclusions: Elevated copeptin levels in patients following cardiac surgery predicted postoperative acute kidney injury development. Therefore, the copeptin concentration after surgery could represent a promising clinical biomarker of the postoperative cardiac outcome.
\end{abstract}

Keywords: Acute kidney injury (AKI); cardiac surgery; copeptin; vasopressin

Submitted Jun 29, 2020. Accepted for publication Sep 26, 2020.

doi: $10.21037 /$ jtd-20-2323

View this article at: http://dx.doi.org/10.21037/jtd-20-2323

\section{Introduction}

Arginine vasopressin (AVP), a neuropeptide stored in the posterior pituitary lobe, maintains the vasomotor tone during shock and promotes vasoconstriction through nonadrenergic pathways (1). Several previous studies showed that AVP levels were associated with heart failure severity (2-4). Despite its clinical importance, it is unclear how endogenous AVP responds to cardiac surgery. In a recent study, AVP plasma concentrations in cardiac surgery patients, $24 \mathrm{~h}$ after intensive care unit (ICU) admission, were higher than those in patients with sepsis or systemic inflammatory response syndrome (5). AVP is highly unstable and rapidly degradable $e x$ vivo even when frozen at $-20{ }^{\circ} \mathrm{C}(6)$. Several studies have suggested that copeptin, the $\mathrm{C}$-terminal portion of the pre-pro-vasopressin molecule, may be a reliable surrogate for AVP (5-7), because it is 
secreted in equimolar amounts to AVP besides being more stable and easier to measure $(8,9)$. Copeptin concentrations in plasma increase as a response to physiological stress and have prognostic value in several disease entities, such as acute myocardial infarction and septic shock $(10,11)$. We hypothesized that copeptin levels in patients admitted to ICU after surgery are mostly affected by surgical stress; thus, the measurement of copeptin levels could enable an early assessment of surgical stress. The present study investigated whether copeptin levels in patients admitted to ICU and following cardiac surgery predicted the clinical outcome of the patients.

Acute kidney injury (AKI) is a common postoperative complication that significantly increases the rates of morbidity and mortality (12-14). AKI has been defined in 2012 by the Kidney Disease Improving Global Outcomes (KDIGO) criteria and is divided into three stages, based on an increase in serum creatinine or urine output (15). Postoperative AKI is a strong predictor of mortality, and severe AKI increases mortality by 3 - to 8 -fold (16). Even a small increase of $0.5 \mathrm{mg} / \mathrm{dL}$ in creatinine levels following cardiac surgery has strongly deleterious effects $(17,18)$. Even though few studies have evaluated postoperative AKI markers, they have not associated them with clinical outcome prediction (19). Therefore, the goal of this study was to determine copeptin levels following surgery and investigate their possible association with postoperative AKI development in a prospective observational cohort of patients undergoing cardiac surgery. We present the following article in accordance with the STROBE reporting checklist (available at http://dx.doi.org/10.21037/jtd-202323).

\section{Methods}

The study was conducted in accordance with the Declaration of Helsinki (as revised in 2013). The study was approved by the Institutional review board of Nara Medical University (December 19th, 2018; approval number: 2050) and informed consent was taken from all the patients.

\section{Study design}

We prospectively enrolled 23 patients aged 18 years or older undergoing cardiac surgery at Nara Medical University Hospital from January 2018 to December 2019 and measured their plasma AVP and copeptin concentrations. Patients with end-stage renal disease on hemodialysis were excluded from the enrollment.

Study patients underwent five blood draws that were performed with arterial catheters or by puncture of a peripheral vein. The first blood draw was performed before or immediately after anesthesia induction in the operating suite. The second blood draw was taken immediately after surgery. The third, fourth, and fifth blood samplings were performed on the first, third, and seventh postoperative days after surgery, respectively. Blood was collected in pre-chilled tubes containing ethylenediamine tetra-acetic acid, and plasma was removed and immediately frozen at $-70{ }^{\circ} \mathrm{C}$ until use. Copeptin levels were measured with a commercially available immunoluminometric assay (B.R.A.H.M.S. LUMItest CT-proAVP, B.R.A.H.M.S. AG, Hennigsdorf/Berlin, Germany). The measurable range of the copeptin assay was $0.7-2,000 \mathrm{pmol} / \mathrm{L}$. Vital signs, blood pressure, and norepinephrine doses were documented. As for the measurement of BNP, blood samples were immediately placed in EDTA-treated tubes, transported to the laboratory department of our hospital, and analyzed by sandwich fluorescence immunoassay (Siemens Healthineers, NY, USA).

\section{Study endpoint}

The primary endpoint was the incidence of postoperative AKI, which was defined according to the 2012 KDIGO criteria: a serum creatinine increase greater than $50 \%$ from baseline or $\geq 0.3 \mathrm{mg} /$ increase within $48 \mathrm{~h}$ (20). The KDIGO urine output criterion was not applied to determine AKI.

\section{Statistical analysis}

Continuous variables were expressed as the means and standard deviations or as medians and interquartile ranges, while categorical variables were presented as numbers and percentages. Comparisons were performed with Student's t-test or the

Wilcoxon rank-sum test for continuous variables and the chi-squared test for categorical variables. Correlations between copeptin levels and variables, such as AVP, were assessed by Bland-Altman analysis, or Pearson's correlation coefficient or Spearman's rank correlation test. According to the median copeptin levels at ICU admission, we set the cut-off serum copeptin level as $43.7 \mathrm{pmol} / \mathrm{L}$ to discriminate high ( $\geq 43.7 \mathrm{pmol} / \mathrm{L})$ - and low $(<43.7 \mathrm{pmol} / \mathrm{L})$-copeptin groups. We estimated the risk of AKI in both groups at ICU admission by the logistic regression model and expressed 
Table 1 Patient characteristics, according to copeptin values at the ICU after surgery

\begin{tabular}{|c|c|c|c|c|}
\hline Characteristics & Study Population $(n=23)$ & $\begin{array}{l}\text { Low-copeptin } \\
(<43.7 \mathrm{pmol} / \mathrm{L})(\mathrm{n}=11)\end{array}$ & $\begin{array}{c}\text { High-copeptin } \\
(\geq 43.7 \mathrm{pmol} / \mathrm{L})(\mathrm{n}=12)\end{array}$ & $P$ value \\
\hline \multicolumn{5}{|l|}{ Baseline clinical data } \\
\hline Age (years) & $74 \pm 6$ & $76 \pm 4$ & $72 \pm 7$ & 0.12 \\
\hline Female gender [\%] & $7[30]$ & $4[36]$ & $3[25]$ & 0.668 \\
\hline NYHA $\geq 3[\%]$ & 9 [39] & $3[27]$ & $6[50]$ & 0.4 \\
\hline \multicolumn{5}{|l|}{ Comorbidities [\%] } \\
\hline Hypertension & $11[48]$ & $4[36]$ & $7[58]$ & 0.4 \\
\hline Diabetes & 8 [35] & $2[18]$ & $6[50]$ & 0.193 \\
\hline CABG & 9 [36] & $3[27]$ & $6[50]$ & 0.4 \\
\hline Valvular surgery & $5[21]$ & $4[36]$ & $1[8]$ & 0.155 \\
\hline $\mathrm{CABG}$ and valvular surgery & $7[30]$ & $3[27]$ & $4[33]$ & 1 \\
\hline Others & $4[17]$ & $1[9]$ & $3[25]$ & 0.59 \\
\hline Operation time (min) & $367 \pm 147$ & $320 \pm 137$ & $410 \pm 148$ & 0.142 \\
\hline Cardiopulmonary bypass time (min) & $197 \pm 104$ & $174 \pm 69$ & $220 \pm 131$ & 0.374 \\
\hline Aortic cross clamp time (min) & $132 \pm 82$ & $108 \pm 42$ & $165 \pm 113$ & 0.282 \\
\hline SVRI on ICU admission & $1967 \pm 509$ & $1859 \pm 403$ & $2066 \pm 591$ & 0.336 \\
\hline Maximum NE dosage (mcg/kg per min) & $0.105 \pm 0.068$ & $0.076 \pm 0.059$ & $0.131 \pm 0.067$ & 0.047 \\
\hline Time on ventilator in ICU (h) & $8[5-15]$ & $8[6-14]$ & $10[5-15]$ & 0.689 \\
\hline ICU LOS (days) & $2[2-4]$ & $2[2-3]$ & $3[2-4]$ & 0.599 \\
\hline
\end{tabular}

ICU, intensive care unit; BSA, body surface area; NYHA, New York Heart Association functional class; LVEF, left ventricular ejection fraction; BNP, B-type natriuretic peptide; CABG, coronary artery bypass grafting; MAP, mean arterial blood pressure; CVP, central venous pressure; Cl, cardiac index; SVRI, systemic vascular resistance index; NE, norepinephrine; LOS, length of stay.

the results as odds ratio (OR) and $95 \%$ confidence intervals (CIs). Differences with two-sided $\mathrm{P}$ values $<0.05$ were statistically significant. JMP software for Windows version 13 (SAS Institute, Cary, NC, USA) was used for all statistical analyses.

\section{Results}

\section{Baseline characteristics}

Table 1 shows patient characteristics, stratified according to low or high copeptin levels following surgery. There was no 
significant difference in age $(\mathrm{P}=0.12)$ or preoperative New York Heart Association functional class $(\mathrm{P}=0.4)$ between the two groups. Additionally, there was no significant difference in preoperative $\mathrm{BNP}(\mathrm{P}=0.48)$, mean arterial pressure $(\mathrm{P}=0.39)$, or any other variable. Although there was no significant difference in ICU length of stay, patients in the high copeptin group required higher norepinephrine doses than those in the low copeptin group.

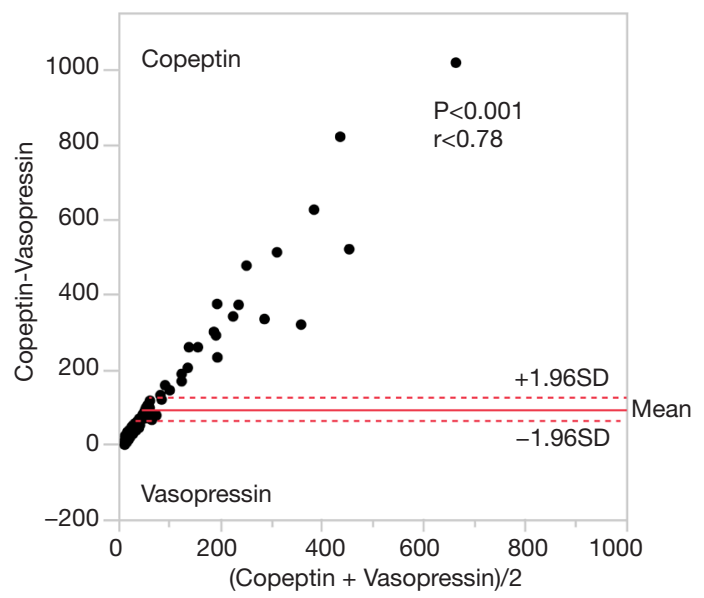

Figure 1 Correlation analysis between copeptin and arginine vasopressin plasma concentrations. A Spearman rank-order correlation test was performed to assess the correlation between copeptin and arginine vasopressin plasma concentrations from 116 pairs of measurements. $\mathrm{r}=0.78, \mathrm{P}<0.001$.

\section{Correlations between copeptin levels and other variables}

Figure 1 shows that AVP and copeptin concentrations were closely correlated $(\mathrm{r}=0.787, \mathrm{P}<0.001)$. The time course of copeptin plasma concentrations in the low and high copeptin groups in our study cohort is shown in Figure 2. Although copeptin concentrations after surgery significantly differed between the two groups $(\mathrm{P}<0.001)$, no differences were observed on the 1 st postoperative day and thereafter. In the low copeptin group, the copeptin concentrations at ICU admission were significantly lower than those on the 1 st postoperative day $(\mathrm{P}<0.001)$, as a difference with the high copeptin group $(\mathrm{P}=0.81)$. Table 2 presents laboratory parameters between low and high copeptin groups during the observational period. AVP and cortisol levels following surgery in the high copeptin group were higher than those in the low copeptin group. Serum creatinine levels on days 1 and 3 after surgery in the high group were higher than those in the low group. No correlation was detected between copeptin concentration and serum osmolarity. Interestingly, central venous pressure (CVP) $(\mathrm{P}=0.03)$ and norepinephrine dose $(\mathrm{P}=0.008)$ were the only parameters significantly correlated with copeptin levels right after surgery, according to the explorative bivariate regression analysis (Figure 3). There was no detectable correlation between copeptin levels and other hemodynamic parameters.

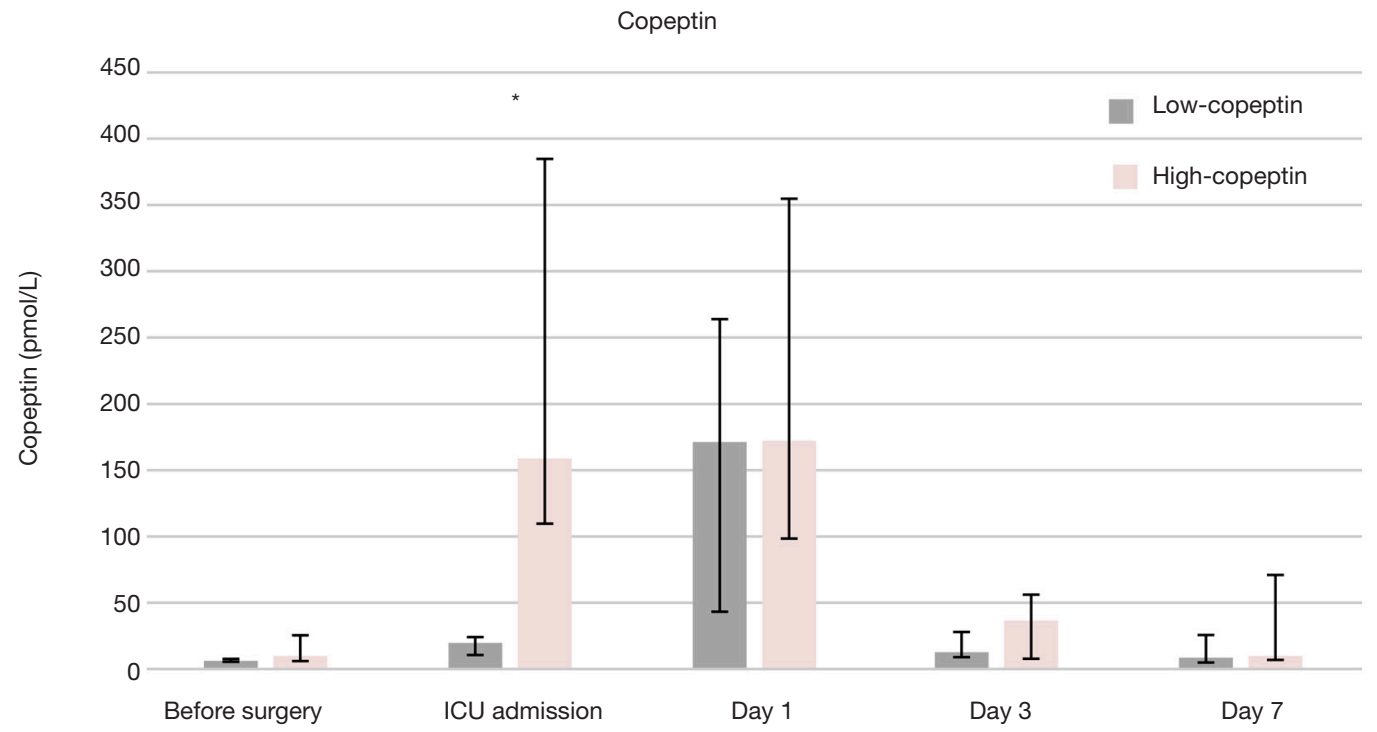

Figure 2 Time course of copeptin plasma concentrations in the low and high copeptin groups in our cohort study. *Significant difference between groups $(\mathrm{P}<0.001)$ 


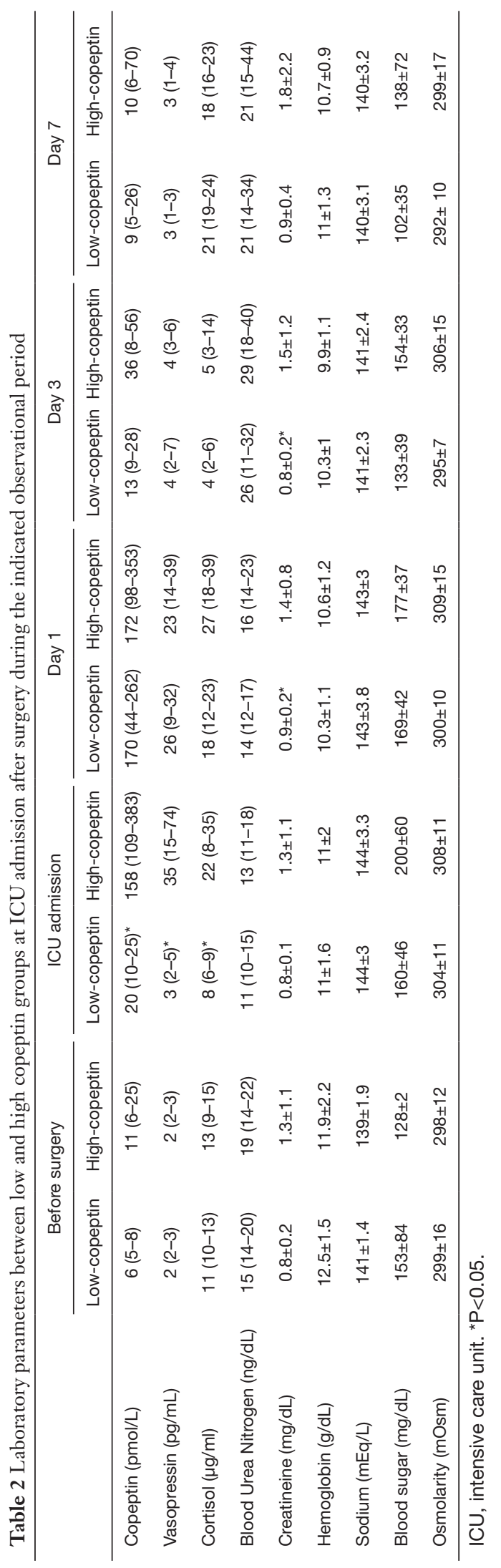

\section{Predictors of AKI}

In this study, six instances of postoperative AKI occurred. Copeptin levels following surgery provided a strong prediction of postoperative AKI (area under the ROC curve of $0.83, \mathrm{P}=0.004$ ), and the cut-off value was $144.5 \mathrm{pmol} / \mathrm{L}$. Results of linear regression followed by a univariate regression are reported in Table 3. Univariate analysis indicated that copeptin levels right after surgery $(\mathrm{P}=0.03)$ and cardiopulmonary bypass time $(\mathrm{P}=0.04)$, without copeptin levels before surgery, as predictors of postoperative AKI.

\section{Discussion}

The principal finding of this study is that copeptin concentration right after surgery is a predictor of postoperative AKI development. Copeptin levels after surgery were also associated with the administered norepinephrine dose, although not with ICU length of stay. To the best of our knowledge, this is the first study to demonstrate an association between copeptin levels following cardiac surgery and clinical outcomes.

Our study evidenced a strong correlation between levels of copeptin and AVP. Interestingly, as shown in Figure 1, the value of copeptin is larger than the value of AVP, and the larger the value of copeptin and AVP, the larger the difference between copeptin and AVP. This finding means that the measured value for larger AVP deviates from the value of copeptin, which is thought to be close to the true value of AVP because AVP and copeptin are produced at equal stoichiometry. Therefore, the larger the AVP value, the more uncertain the AVP measurement. This observation suggests that copeptin needs to be measured to study the role of AVP in cardiovascular surgery and that under high levels of stress, such as undergoing cardiac surgery, copeptin levels may reflect the real degree of the stress rather than that approximated by the AVP levels. As a result, it might be natural that copeptin as a substitute for AVP should indicate the degree of the impact of AVP on the renal function, considering the kidney is one of the main target organs of AVP under the arterial underfilling condition such as following cardiac surgery. However, more comprehensive studies to unravel copeptin's predictive role in terms of the true AVP value in cardiac surgery should be carried out soon. Furthermore, we investigated the copeptin concentration following surgery as a novel biomarker for hemodynamic stress. Although several previous studies 
A

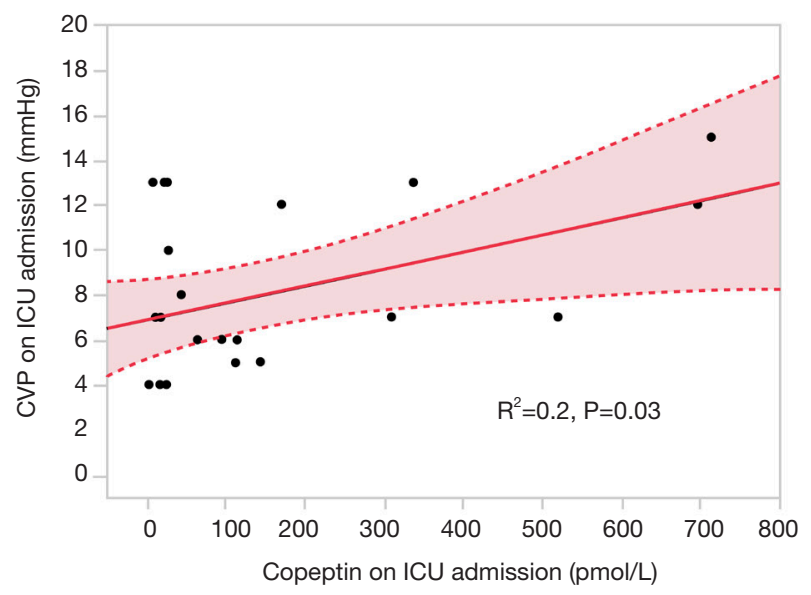

B

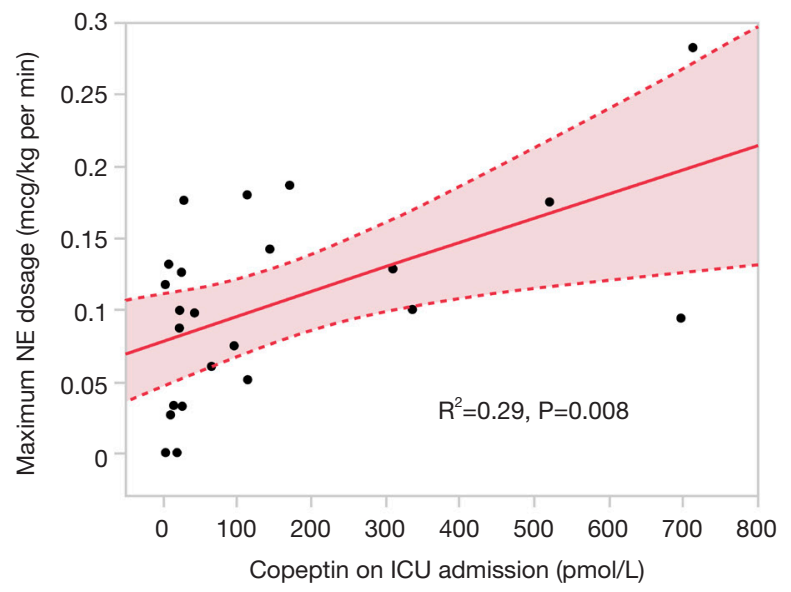

Figure 3 Correlation of copeptin levels right after surgery with (A) central venous pressure (CVP) and (B) maximum norepinephrine dose.

Table 3 Univariate predictors of postoperative AKI

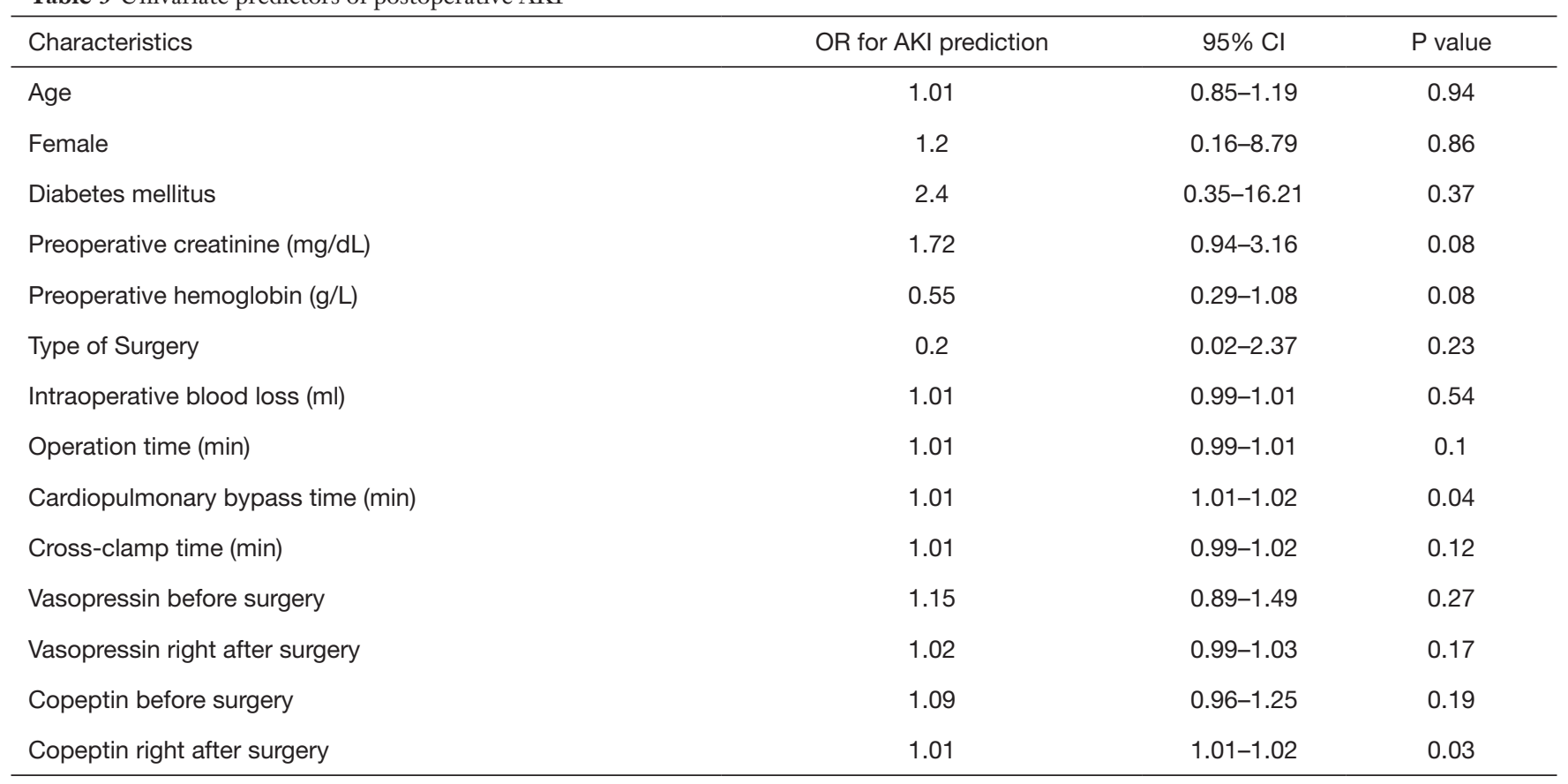

$\mathrm{AKI}$, acute kidney injury; OR, odds ratio; $\mathrm{Cl}$, confidence interval; ICU, intensive care unit.

have reported an association between copeptin levels before and one day after surgery with clinical outcome $(21,22)$, our study of copeptin levels immediately after surgery was specifically assessed as a biomarker for surgical stress. As Table 3 shows, our analysis of predictors of postoperative AKI seems to indicate that copeptin right after surgery may have more clinical significance than those in another period.
Further studies on the clinical significance of copeptin levels immediately after cardiac surgery should be performed in the future. Among all the studied parameters, a strong association was detected only between copeptin levels and CVP following surgery. Spiegel documented that although the physiology behind venous return is extremely complex, operative stress can reduce the vessel wall compliance (with 
the addition of vasopressor agents), changing the venous return (23). Our results suggested that surgical stress may influence the physiology of venous return. In our cohort, these hemodynamic changes, including in CVP levels, may have influenced the development of postoperative AKI.

Postoperative AKI is a relatively common complication of cardiothoracic surgery and has short- and long-term survival implications, even for those who do not progress to renal failure. Currently, the incidence of renal failure onset that requires dialysis is approximately $2 \%$ (24). Additionally, as there are no active treatments for AKI, clinicians focus on prevention and risk factor management. In-depth knowledge of the risk factors and pathogenesis associated with AKI offers some guidance to clinicians for the prevention and management of AKI. Postoperatively, urine IL-18, and plasma neutrophil gelatinase-associated lipocalin (NGAL) levels peaked within 6 hours after ICU admission and were strongly associated with AKI (25). Although several postoperative biomarkers can predict the risk and enable early diagnosis of AKI in patients undergoing cardiac surgery (26-28), additional assessments are required to determine their potential to predict improved outcomes. In recent years, there have been several reports that biomarkers associated with surgical and kidney stress are important in predicting postoperative AKI after cardiac surgery (29-32). Therefore, the discovery that copeptin levels immediately after surgery predicted the postoperative AKI condition was of great clinical importance. However, the implications between this association and the subsequent clinical outcomes require more research. Several studies showed that combined surgical procedures (valve replacement and coronary artery bypass) and prolonged cardiopulmonary bypass times increased the incidence of AKI $(33,34)$. Cardiopulmonary bypass times and copeptin levels following surgery were also predictors of postoperative AKI in our study. We believe that the stress generated due to cardiac surgery, including the use of cardiopulmonary bypass, affected copeptin levels immediately after surgery, and those subsequent hemodynamic changes led to postoperative AKI onset. Thus, copeptin levels right after surgery could reflect the overall endogenous stress of the patient, predicting mortality, independently of the underlying pathology.

\section{Study limitations}

This study has several limitations. First, this was a singlecenter study that only included 23 patients, who were followed for a short period, resulting in a relatively small number of postoperative AKI, and thus of few covariates that were only analyzed with univariate models. However, follow-up was complete for all patients, and patients who had end-stage renal disease with hemodialysis were excluded because of the presence of a variety of confounding factors. The fact that copeptin levels at ICU admission predicted adverse events even in a relatively small sample size highlighted the strong correlation between those parameters. Finally, several surgeons were involved during this period, which could increase the variability of the data; however, the performance of the surgeon may not considerably affect the outcomes.

\section{Conclusions}

Although the use of copeptin concentration as a novel risk factor for cardiac surgery should be further validated.; we provided evidence that copeptin concentration serves as a biomarker of postoperative AKI onset after cardiac surgery.

\section{Acknowledgments}

Funding: None.

\section{Footnote}

Reporting Checklist: The authors have completed the STROBE reporting checklist. Available at http://dx.doi. org/10.21037/jtd-20-2323

Data Sharing Statement: Available at http://dx.doi. org/10.21037/jtd-20-2323

Conflicts of Interest: All authors have completed the ICMJE uniform disclosure form (available at http://dx.doi. org/10.21037/jtd-20-2323). The authors have no conflicts of interest to declare.

Ethical Statement: The authors are accountable for all aspects of the work in ensuring that questions related to the accuracy or integrity of any part of the work are appropriately investigated and resolved. The study was conducted in accordance with the Declaration of Helsinki (as revised in 2013). The study was approved by the Institutional review board of Nara Medical University (December 19th, 2018; approval number: 2050) and informed consent was taken from all the patients. 
Open Access Statement: This is an Open Access article distributed in accordance with the Creative Commons Attribution-NonCommercial-NoDerivs 4.0 International License (CC BY-NC-ND 4.0), which permits the noncommercial replication and distribution of the article with the strict proviso that no changes or edits are made and the original work is properly cited (including links to both the formal publication through the relevant DOI and the license). See: https://creativecommons.org/licenses/by-nc-nd/4.0/.

\section{References}

1. Oliver JA, Landry DW. Endogenous and exogenous vasopressin in shock. Curr Opin Crit Care 2007;13:376-82.

2. Francis GS, Benedict C, Johnstone DE, et al. Comparison of neuroendocrine activation in patients with left ventricular dysfunction with and without congestive heart failure. A substudy of the Studies of Left Ventricular Dysfunction (SOLVD). Circulation 1990;82:1724-9.

3. Rouleau JL, Packer M, Moyé L, et al. Prognostic value of neurohumoral activation in patients with an acute myocardial infarction: effect of captopril. J Am Coll Cardiol 1994;24:583-91.

4. Nakamura T, Funayama H, Yoshimura A, et al. Possible vascular role of increased plasma arginine vasopressin in congestive heart failure. Int J Cardiol 2006;106:191-5.

5. Jochberger S, Mayr VD, Luckner G, et al. Serum vasopressin concentrations in critically ill patients. Crit Care Med 2006;34:293-9.

6. Morgenthaler NG, Struck J, Alonso C, et al. Assay for the measurement of copeptin, a stable peptide derived from the precursor of vasopressin. Clin Chem 2006;52:112-9.

7. Szinnai G, Morgenthaler NG, Bernis K, et al. Changes in plasma copeptin, the $\mathrm{C}$-terminal portion of arginine vasopressin during water deprivation and excess in healthy subjects. J Clin Endocrinol Metab 2007;92:3973-8.

8. Schurtz G, Lambin N, Bauters C, et al. Copeptin in acute coronary syndromes and heart failure management: state of the art and future directions. Arch Cardiovasc Dis 2015;108:398-407.

9. Balling L, Gustafsson F. Copeptin as a biomarker in heart failure. Biomark Med 2014;8:841-54.

10. Lee JH, Chan YH, Lai OF, et al. Vasopressin and copeptin levels in children with sepsis and septic shock. Intensive Care Med 2013;39:747-53.

11. Möckel M, Searle J. The positive predictive value of ct-proAVP (copeptin) in patients with STEMI. Heart 2013;99:1475.
12. Crawford TC, Magruder JT, Grimm JC, et al. Renal failure after cardiac operations: not all acute kidney injury is the same. Ann Thorac Surg 2017;104:760-6.

13. Xu JR, Zhu JM, Jiang J, et al. Risk factors for long-term mortality and progressive chronic kidney disease associated with acute kidney injury after cardiac surgery. Medicine (Baltimore) 2015;94:e2025.

14. Thongprayoon C, Cheungpasitporn W, Shah IK, et al. Long-term outcomes and prognostic factors for patients requiring renal replacement therapy after cardiac surgery. Mayo Clin Proc 2015;90:857-64.

15. Kellum JA, Lameire N. Diagnosis, evaluation, and management of acute kidney injury: a KDIGO summary (part 1). Crit Care 2013;17:204.

16. Ortega-Loubon C, Fernández-Molina M, CarrascalHinojal Y, et al. Cardiac surgery-associated acute kidney injury. Ann Card Anaesth 2016;19:687-98.

17. Chertow GM, Levy EM, Hammermeister KE, et al. Independent association between acute renal failure and mortality following cardiac surgery. Am J Med 1998;104:343-8.

18. Ho J, Tangri N, Komenda P, et al. Urinary, plasma, and serum biomarkers' utility for predicting acute kidney injury associated with cardiac surgery in adults: a metaanalysis. Am J Kidney Dis 2015;66:993-1005.

19. Howitt SH, Grant SW, Caiado C, et al. The KDIGO acute kidney injury guidelines for cardiac surgery patients in critical care: a validation study. BMC Nephrol 2018;19:149.

20. Kiers HD, van den Boogaard M, Schoenmakers MC, et al. Comparison and clinical suitability of eight prediction models for cardiac surgery-related acute kidney injury. Nephrol Dial Transplant 2013;28:345-51.

21. Colson PH, Bernard C, Struck J, et al. Post cardiac surgery vasoplegia is associated with high preoperative copeptin plasma concentration. Crit Care 2011;15:R255.

22. Schrimpf C, Gillmann HJ, Sahlmann B, et al. Renal function interferes with copeptin in prediction of major adverse cardiac events in patients undergoing vascular surgery. PLoS One 2015;10:e0123093.

23. Spiegel R. Stressed vs. unstressed volume and its relevance to critical care practitioners. Clin Exp Emerg Med 2016;3:52-4.

24. Koyner JL, Garg AX, Coca SG, et al. Biomarkers predict progression of acute kidney injury after cardiac surgery. J Am Soc Nephrol 2012;23:905-14.

25. Kuitunen A, Vento A, Suojaranta-Ylinen R, et al. Acuterenal failure after cardiac surgery: Evaluation of the 
RIFLE classification. Ann Thorac Surg 2006;81:542-6.

26. Lee CC, Chang CH, Cheng YL, et al. Diagnostic Performance of Cyclophilin A in Cardiac SurgeryAssociated Acute Kidney Injury. J Clin Med 2019:9:108.

27. Du S, Tian J, Xiao Z, et al. Serum alpha 1-antitrypsin predicts severe acute kidney injury after cardiac surgery. J Thorac Dis 2019;11:5053-62.

28. Moledina DG, Mansour SG, Jia Y, et al. Association of T cell-derived inflammatory cytokines with acute kidney injury and mortality after cardiac surgery. Kidney Int Rep 2019;4:1689-97.

29. Kararmaz A, Arslantas MK, Aksu U, et al. Evaluation of acute kidney injury with oxidative stress biomarkers and Renal Resistive Index after cardiac surgery. Acta Chir Belg 2019;13:1-9.

30. Engelman DT, Crisafi C, Germain M, et al. Using urinary

Cite this article as: Yamashita K, Abe T, Hayata Y, Hirose T, Hiraga S, Fukuba R, Takemura J, Tonomura R, Yamamoto K, Yokoyama S, Taniguchi S. Copeptin concentration following cardiac surgery as a prognostic marker of postoperative acute kidney injury: a prospective cohort study. J Thorac Dis 2020;12(11):6609-6617. doi: 10.21037/jtd-20-2323 biomarkers to reduce acute kidney injury following cardiac surgery. J Thorac Cardiovasc Surg 2020;160:1235-46.e2.

31. Averdunk L, Fitzner C, Levkovich T, et al. Secretory leukocyte protease inhibitor (slpi)-a novel predictive biomarker of acute kidney injury after cardiac surgery: a prospective observational study. J Clin Med 2019;8:1931.

32. Neyra JA, Hu MC, Minhajuddin A, et al. Kidney Tubular Damage and Functional Biomarkers in Acute Kidney Injury Following Cardiac Surgery. Kidney Int Rep 2019;4:1131-42.

33. Rosner MH, Portilla D, Okusa MD. Cardiac surgery as a cause of acute kidney injury: Pathogenesis and potential therapies. J Intensive Care Med 2008;23:3-18.

34. Fischer UM, Weissenberger WK, Warters RD, et al. Impact of cardiopulmonary bypass management on postcardiac surgery renal function. Perfusion 2002;17:401-6. 